\title{
Depressive symptom in the first-episode schizophrenic patients is related to the reduction of grey matter volume in the subgenual anterior cingulate cortex - a pilot study
}

\author{
Jiaojiao Xu ${ }^{1, *}, X^{1}$ ibo He ${ }^{2,3, *}$, Ce Chen ${ }^{4, *}$, Hongjun Tian ${ }^{5}$, Lina Wang ${ }^{5}$, Jie Li ${ }^{5}$, Fuqiang \\ $\mathrm{MaO}^{2}$, Chuanjun Zhuo ${ }^{2,4,5}$ and Danmin Miao ${ }^{1}$ \\ ${ }^{1}$ Faculty of Medical Psychology, The Fourth Military Medical University, Xian, Shanxi, China \\ ${ }^{2}$ The Department of Psychiatry, Tianjin Medical University, Tianjin, China \\ ${ }^{3}$ The Department of Psychiatry, Tianjin Anding Hospital, Tianjin, China \\ ${ }^{4}$ The Department of Psychiatry, Wenzhou Seventh People's Hospital, Wenzhou, Zhejiang, China \\ ${ }^{5}$ The Department of Psychiatric Neuroimaging Laboratory, Tianjin Anding Hospital, Tianjin, China \\ *These authors contributed equally to this work
}

Correspondence to: Chuanjun Zhuo, email: chuanjunzhuotjmh@163.com, jshkhl@126.com

Danmin Miao, email: miaodanmin@126.com

Keywords: first episode schizophrenia; depressive symptom; grey matter volume; MRI; sgACC

Received: September 26, 2017

Accepted: December 04, 2017

Published: January 12, 2018

Copyright: Xu et al. This is an open-access article distributed under the terms of the Creative Commons Attribution License 3.0 (CC BY 3.0), which permits unrestricted use, distribution, and reproduction in any medium, provided the original author and source are credited.

\section{ABSTRACT}

Approximately $25-75 \%$ of patients with schizophrenia also present major depressive systems. Investigations into the pathological features associated with depressive symptoms in patients with schizophrenia would provide important information for the development of treatments for depressive symptoms in patients with schizophrenia. In present study, we enrolled 17 first-episode schizophrenic patients with depressive symptoms and 17 well-matched first-episode schizophrenic patients without depressive symptoms and compared differences in their grey mater volume (GMV) using voxel-based morphometry (VBM). We observed significantly decreased GMV in the subgenual anterior cingulate cortex (sgACC) in the first episode schizophrenic patients with depressive symptoms compared to the first episode schizophrenic patients without depressive symptoms. However, we did not observe a correlation between the severity of the decreased GMV and the severity of depressive symptoms. Although convincing evidence supporting this postulation is lacking and requires further study for confirmation, to some extent, our findings provide indirect evidence for the suggestion that the reduction of the GMV in the sgACC may play a key role in the depressive symptoms in the first episode schizophrenic patients.

\section{INTRODUCTION}

According to several previous studies, depressive symptoms are usually accompanied with psychotic disorders, particularly schizophrenia $[1,2]$. Most notably, depressive symptoms could occur in every stage of schizophrenia, including the prodromal stage, acute episode stage and remission stage. Many previous studies have confirmed that approximately $25-75 \%$ of patients with schizophrenia also demonstrate with different levels of depressive symptoms $[3,4]$. Depressive symptoms in patients with schizophrenia are usually induced by detrimental consequences, including suicidal ideations, an increased relapse rate and poor quality of life. The above-mentioned detrimental consequences directly increase mortality of patients with schizophrenia $[5,6]$. Hence, explorations of the brain-related pathological features of depressive symptoms in patients with schizophrenia and the specific treatment targets have become the key issues in developing effective treatment strategies for depressive symptoms in the schizophrenic patients, especially in the first episode schizophrenic patients with depressive symptoms [8].

Based on the advances in neuroimaging techniques in the last two decades, many studies have adopted magnetic resonance imaging (MRI) and functional MRI 
(fMRI) techniques to explore the pathological features of major depression disorder (MDD) and schizophrenia. These studies have acquired important and useful information that has improved our understanding of the biological basis of schizophrenia and have provided many indices to aid psychiatrists in improving the rates of accurate diagnoses and treatment efficacies [9-17]. However, most of these previous studies have focused on exploring the common brain features shared by patients with schizophrenia and depression, these previous studies have provided evidence that structural or functional impairments in some brain regions in patients with schizophrenia overlap with impairments observed in patients with MDD to some extent, The temporal lobe and anterior cingulate are the main affected regions [7, 18-31].

Although previous studies have provided important and useful information that has improved our understanding of the association of depressive symptoms with pathological characteristics in the brains of patients with schizophrenia, to the best of our knowledge, few studies have reported the association of depressive symptoms with structural alterations in the brains of patients with first-episode schizophrenia. In the present study, our aims were to compare the alterations in grey matter volume (GMV) between patients with first-episode schizophrenia patients who accompanied with and without depressive symptoms. We assumed that the firstepisode schizophrenic patients with depressive symptoms probably had specific structural alterations compared to the first episode schizophrenic patients without depressive symptoms.

\section{RESULTS}

\section{Demographics and clinical characteristics}

The demographic and clinical characteristics of the participants are presented in Table 1 . No significant group differences were observed in gender $\left(X^{2}=-0.806\right.$, $P=0.426)$, age $(\mathrm{t}=-0.497, P=0.623)$, educational level $(\mathrm{t}=0.539, P=0.534)$, illness duration $(\mathrm{t}=-0.331$, $P=0.743)$, antipsychotic drug dosages $(\mathrm{t}=0.484$, $P=0.631)$, or PANSS scores $(\mathrm{t}=0.094, P=0.926)$. The mean antipsychotic dosages (chlorpromazine equivalents) were $533.0 \pm 78.7 \mathrm{mg} / \mathrm{d}$ in the patients with depressive symptoms and $526.6 \pm 79.8 \mathrm{mg} / \mathrm{d}$ in the patients without depressive symptoms. Fourteen patients with depressive symptoms were administered five antidepressants, including venlafaxine, mirtazapine, citalopram, sertraline, and fluoxetine, and three patients were simultaneously treated with mirtazapine and venlafaxine (i.e., the "California rocket treatment method") for very severe depression. Detailed information about the demographic and clinical characteristics is listed in Table 1.

\section{GMV alterations in first episode schizophrenic patients with moderate to severe depressive symptoms}

Compared to first-episode schizophrenic patients without depressive symptoms, decreased GMV were observed in the subgenual anterior cingulate cortex (sgACC) and bilateral occipital lobe in the first-episode schizophrenic patients with moderate to severe depressive symptoms. However, after the false discovery rate (FDR) correction, only the reduced GMV in the sgACC was significant (Figure 1). The difference in the GMV in the bilateral occipital lobe disappeared (Figure 2). Additionally, the partial correlation analysis did not find any correlations between the severity of the GMV reduction and the severity of the CDSS scores.

\section{DISCUSSION}

In the present study, we initially compared the differences in GMV between the first-episode schizophrenic patients who accompanied with and without depressive symptoms. GMV was decreased in the sgACC in the first-episode schizophrenic patients who accompanied with depressive symptoms. Thus, the decreased GMV in the sgACC might be a specific pathological feature of depressive symptoms in patients with first-episode schizophrenia. Many previous studies have focused on investigating the pathological features of depression and confirmed that structural and functional alterations in the sgACC are pathological features of patients with depression. Moreover, these alterations are also related to the treatment response, particularly in patients with treatment-refractory depression [32-36]. Simultaneously, according to some previous studies, structural and functional alterations in the temporal lobe and sgACC overlap in patients with major depression and schizophrenia [8, 9, 10,37]. Our findings converged with the previous findings to support the hypothesis that the sgACC is a pathological centre in patients with MDD and the hypothesis that the sgACC is a common pathological feature in patients with schizophrenia and depression [32-37]. To some extent, our findings provide indirect evidence for the postulation that the sgACC may be one of the specific pathological features of depressive symptoms in patients with schizophrenia, although conclusive evidence supporting this postulate is lacking and requires further study for confirmation.

The severity of depressive symptoms in patients with schizophrenia differs in different illness stages, and more severe depressive symptoms usually occur in the first-episode stage [38]. The psychological explanation for this phenomenon is that it may be a natural reaction to the development of psychotic symptoms [39]. According to this postulate, psychotic symptoms cause psychological stress, which may be the reason for the 
severe depressive symptoms. Chronic stress has been shown to cause depressive reactions and reduced GMV in some but not all patients. The patients in the present study experienced illness at a mean of 7.5 months, which is sufficient period to cause GMV reductions. Our findings support the hypothesis that the psychiatric symptoms induced psychological stress, subsequently induced the GMV reduction in some key brain regions, such as sgACC. [40, 41].

A counterintuitive phenomenon observed in this study is the lack of correlation between the severity of the GMV reduction and the severity of depressive symptoms. However, this counterintuitive phenomenon is not incredible; in several of our previous studies, we did not observe correlations between the GMV alterations and PANSS scores, illness duration, or dosage of antipsychotics. Some other previous studies have also reported this counterintuitive phenomenon. Previous studies have proposed that the severity of structural or functional alterations does not correlate with the severity of clinical symptoms, possibly indicating that the structural or functional alterations should only be used as a quality index to assist in the disease diagnosis and not as a quantity index to assess the severity of the disease [41-44].

\section{Limitations}

The present study has several limitations. The first limitation is that limited by the previous designed cohort study's deficit, we did not find well-matched healthy controls for enrolment in the study; hence, we could not adopt one-way analysis of covariance (ANCOVA) methods to compare the differences in GMV between the two patient groups and healthy controls. Hence, our current conclusion is not convincing. Second, in the present study, patients with first episode schizophrenia patients who accompanied with depressive symptoms were administered antidepressants, and patients without depressive symptoms did not receive antidepressant treatment; thus, the antidepressants may have influenced the findings. Due to a lack of a uniform equation to convert the dosages of antidepressants to a standard similar to the antipsychotics, we could not regress out the influences of the antidepressants using the same method as for the antipsychotics. These influences were not examined under these conditions. Fortunately, in many previous studies, antidepressant dosages were normalized to GMV impairment. These reports provide indirect support for our hypothesis.

\section{MATERIALS AND METHODS}

\section{Sample}

The present study included 17 first-episode schizophrenic patients who accompanied with moderate to severe depressive symptoms and 17 well-matched firstepisode schizophrenic patients who did not accompany with depressive symptoms. The entire sample was derived from our research database of hospitalized patients at Tianjin Mental Health Center, and healthy volunteers were recruited through advertisements. The diagnoses of patients with first-episode schizophrenia patients who accompanied with and without depressive symptoms were determined by two professional psychiatrists who

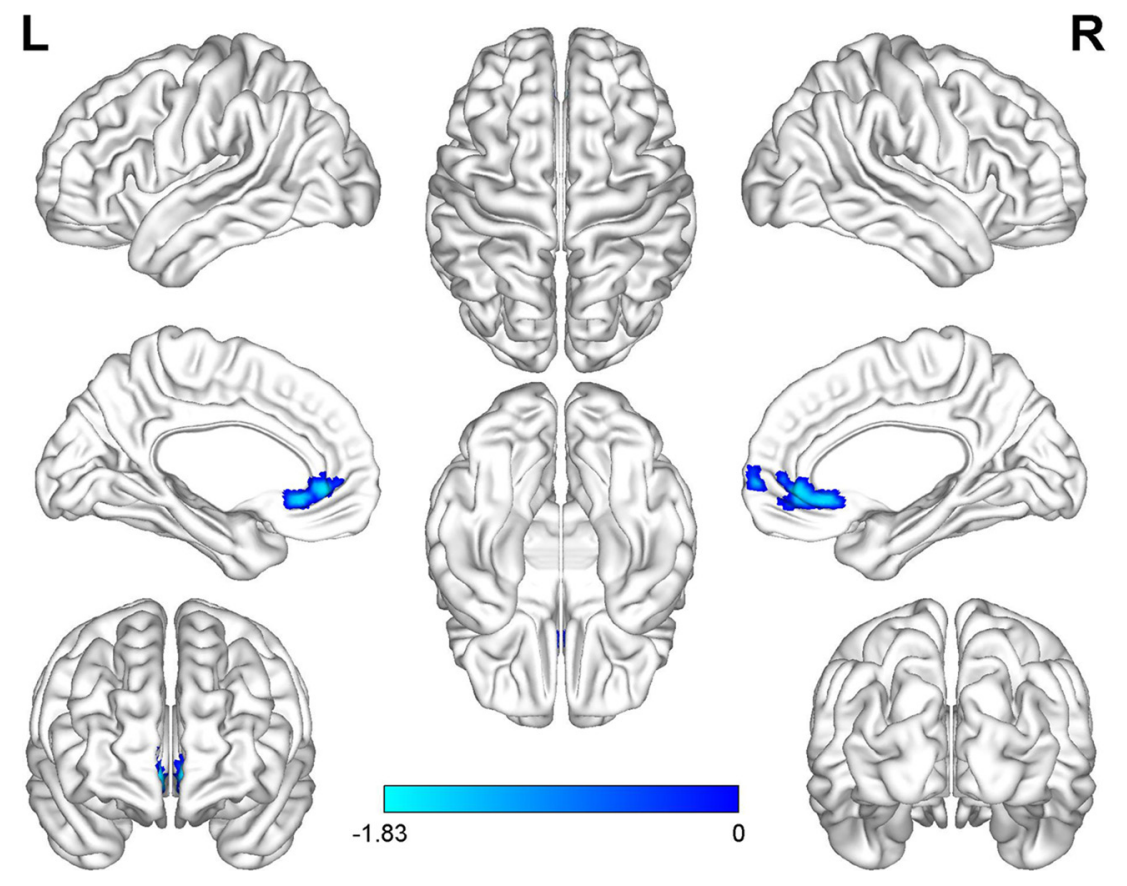

Figure 1: Decreased GMV in the sgACC (after the FDR correction). 
Table 1: Demographic and clinical characteristics

\begin{tabular}{lcccc}
\hline Characteristics & FSC + D & FSC & $\boldsymbol{T} / \boldsymbol{X}^{2}$ & $\boldsymbol{P}$ value \\
\hline Age & $27.1 \pm 5.3$ & $27.6 \pm 5.1$ & -0.497 & 0.623 \\
Gender (male/female) & $10 / 7$ & $8 / 9$ & -0.806 & 0.426 \\
Education level & $12.3 \pm 2.7$ & $12.0 \pm 2.6$ & 0.539 & 0.534 \\
PANSS scores & $140 \pm 21.6$ & $139.7 \pm 21.0$ & 0.094 & 0.926 \\
Illness duration (months) & $3.3 \pm 1.5$ & $3.3 \pm 1.6$ & -0.331 & 0.743 \\
Chlorpromazine equivalent dosage & $533.0 \pm 78.7$ & $526.6 \pm 79.8$ & 0.484 & 0.631 \\
CDSS score & $16.8 \pm 3.0$ & $\mathrm{NA}$ & & \\
\hline
\end{tabular}

Abbreviations: FSC + D: First-episode schizophrenic patients accompanied with depressive symptoms; FSC: First-episode schizophrenic patients without depressive symptoms.

adopted the Structured Clinical Interview for Diagnostic and Statistical Manual of Mental Disorders IV (DSMIV) Disorders (SCID) [45]. In the present study, the first episode was referred as that the illness duration of the schizophrenic patients did not exceed 6 months. The exclusion criteria for all samples were: 1) a history of consciousness disturbances lasting more than $5 \mathrm{~min}$; 2) a history of substance abuse; 3 ) female patients who were pregnant and lactating; 4) a history of any physical and neurological illnesses, such as cardiovascular disease and epilepsy; and 5) any contraindications for MRI scanning. Schizophrenic symptoms were assessed with the Positive and Negative Syndrome Scale (PANSS) [46]. Depressive symptoms were assessed with the Calgary Depression Rating Scale (CDSS) [47], which is usually adopted to assess the severity of depressive symptoms in patients with schizophrenia. For each patient, the chlorpromazine equivalent dose was calculated according to the antipsychotic drugs and dosages used in the last week before the MRI scan, according to uniform criteria [48]. However, due to the lack of uniform criteria for calculating the equivalent dosages of antidepressants, a uniform dosage of the antidepressants used by the patients was not calculated. The Medical Research Ethics Committee of Tianjin Anding Mental Health Center approved our study. The patients' guardians and healthy participants all completely understood the risks and benefits of the study, and written informed consent was obtained from each of the subjects.

\section{MRI data acquisition}

MRI images were acquired with a 3.0-Tesla MR system (Discovery MR750, General Electric, Milwaukee, WI, USA). Foam padding was used to minimize head motion, and earplugs were used to minimize scanner

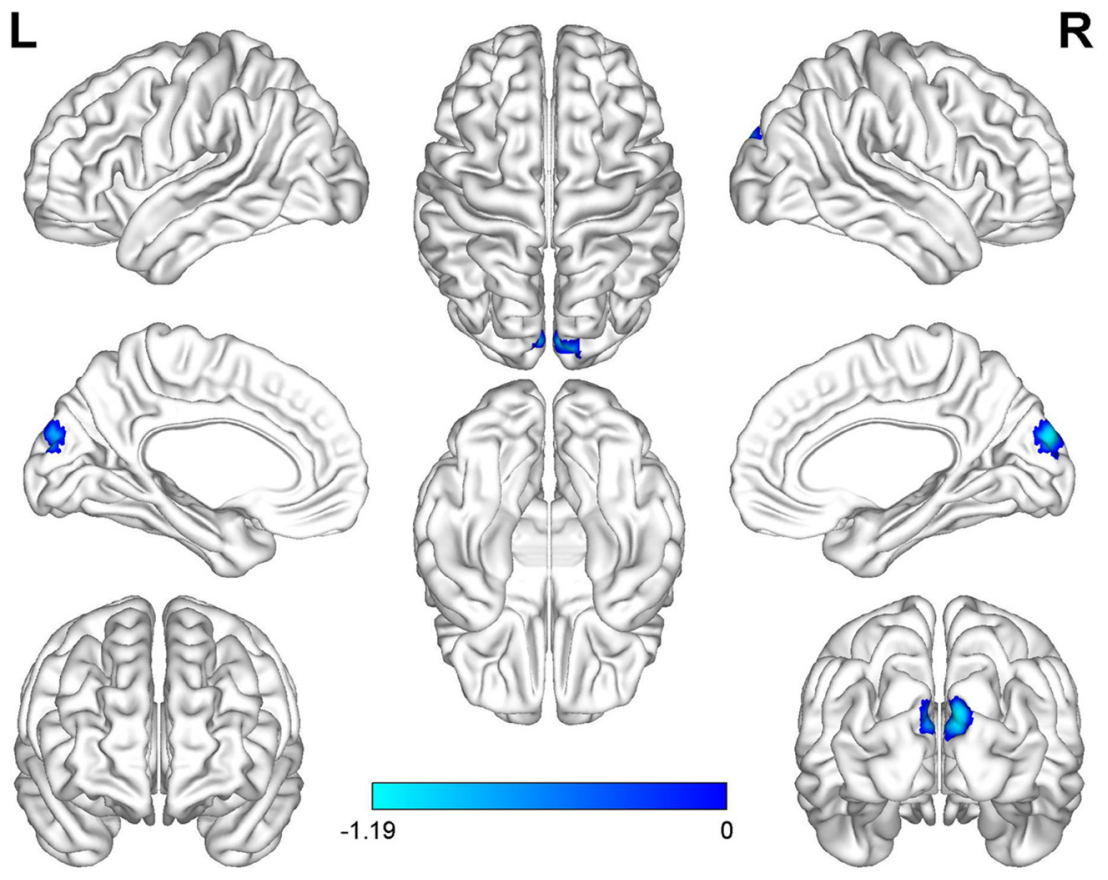

Figure 2: Decreased GMV in the occipital lobe (before the FDR correction). 
noise. Sagittal 3D T1-weighted images were obtained with a brain volume sequence using the following parameters: repetition time $(\mathrm{TR})=8.2 \mathrm{~ms}$; echo time $(\mathrm{TE})=3.2 \mathrm{~ms}$; inversion time $(\mathrm{TI})=450 \mathrm{~ms}$; flip angle $(\mathrm{FA})=12^{\circ}$; matrix $=256 \times 256$; field of view $(\mathrm{FOV})=256 \mathrm{~mm} \times 256$ $\mathrm{mm}$; slice thickness $=1 \mathrm{~mm}$; no gap; and 188 sagittal slices. During the scanning period, all subjects were asked to keep their eyes closed, relax, control their head movement, and think of nothing while not falling asleep.

\section{GMV calculation}

SPM8 (http://www.fil.ion.ucl.ac.uk/spm/software/ spm8/) software was adopted to calculate the GMV of each voxel. According to the operational manual, the structural MR images were segmented into grey matter (GM), white matter and cerebrospinal fluid (CSF) using the standard unified segmentation model. When an initial affine registration of the GM concentration map into the Montreal Neurological Institute (MNI) space was performed, the GM concentration images were nonlinearly warped by diffeomorphic anatomical registration using the exponentiated lie algebra technique, and the results were resampled to a voxel size of $3 \mathrm{~mm} \times 3 \mathrm{~mm} \times 3 \mathrm{~mm}$. The GMV of each voxel was acquired by multiplying the GM concentration map by the non-linear determinants extracted from the spatial normalization step. A full-width at half-maximum Gaussian kernel of $6 \mathrm{~mm} \times 6 \mathrm{~mm} \times 6 \mathrm{~mm}$ was adopted to smooth the GMV images. After spatial preprocessing, the smoothed GMV maps were used for the statistical analyses.

\section{Statistical analysis}

Group differences in the GMVs were compared in a voxel-wise manner using a two-sample $t$-test, with age and gender as nuisance variables. Multiple comparisons were corrected using a false discovery rate method with a significant threshold of $P<0.05$. Partial correlation analyses were used to test the relationship between the GMV and the CDSS scores. Age, gender, antipsychotic dosage, duration of illness and PANSS scores were regressed out, and multiple comparisons were corrected using the Bonferroni method $(P<0.05)$.

\section{CONCLUSIONS}

Our present study is the first to find that the GMV in the sgACC was significantly reduced in the firstepisode schizophrenic patients who accompanied with moderate to severe depressive symptoms. This finding provides evidence to support the hypotheses that structural alterations in the sgACC are pathological features of MDD and that the sgACC is a common pathological feature of schizophrenia and depression. To some extent, our findings also provide indirect evidence for the suggestion that the
sgACC impairment may play key role in the depressive symptoms in the first-episode schizophrenic patients, although convincing evidence supporting this postulation is lacking and requires further study for confirmation.

\section{Author contributions}

$\mathrm{CZ}$ and DM: conceptual design and writing of the draft manuscript; CC, CS,RJ and WZ: conceptual design and writing of the final manuscript; CC,LW, XH and DJ collected and analyzed the data enrolled in this manuscript.

\section{ACKNOWLEDGMENTS AND FUNDING}

This work was supported by a grant from the Tianjin Health Bureau Foundation (2014KR02 to C.Z.), Key Projects of Natural Science Foundation of Tianjin, China(17JCZDJC35700 to C.Z.) , Projects of Natural Science Foundation of Tianjin, ( 16JCYBJC24200 to J.L.) and Tianjin Health Key Program (13KG118 to J.L).

\section{CONFLICTS OF INTEREST}

The authors declare no conflicts of interests.

\section{REFERENCES}

1. Zisook S, McAdams LA, Kuck J, Harris MJ, Bailey A, Patterson TL, Judd LL, Jeste DV. Depressive symptoms in schizophrenia. Am J Psychiatry. 1999; 156:1736-43.

2. Zisook S, Nyer M, Kasckow J, Golshan S, Lehman D, Montross L. Depressive symptom patterns in patients with chronic schizophrenia and subsyndromal depression. Schizophr Res. 2006; 86:226-33.

3. Buckley PF, Miller BJ, Lehrer DS, Castle DJ. Psychiatric comorbidities and schizophrenia. Schizophr Bull. 2009; 35:383-402.

4. Birchwood M, Iqbal Z, Upthegrove R. Psychological pathways to depression in schizophrenia: studies in acute psychosis, post psychotic depression and auditory hallucinations. Eur Arch Psychiatry Clin Neurosci. 2005; 255:202-12.

5. Rocca P, Bellino S, Calvarese P, Marchiaro L, Patria L, Rasetti R, Bogetto F. Depressive and negative symptoms in schizophrenia: different effects on clinical features. Compr Psychiatry. 2005; 46:304-10.

6. Addington DE, Addington JM. Attempted suicide and depression in schizophrenia. Acta Psychiatr Scand. 1992; 85:288-91.

7. Kumari V, Peters E, Guinn A, Fannon D, Russell T, Sumich A, Kuipers E, Williams SC, Ffytche DH. Mapping Depression in Schizophrenia: A Functional Magnetic Resonance Imaging Study. Schizophr Bull. 2016; 42:802-13.

8. Stuhrmann A, Suslow T, Dannlowski U. Facial emotion processing in major depression: a systematic review of 
neuroimaging findings. Biol Mood Anxiety Disord. 2011; 1:10.

9. Sheline YI, Barch DM, Donnelly JM, Ollinger JM, Snyder AZ, Mintun MA. Increased amygdala response to masked emotional faces in depressed subjects resolves with antidepressant treatment: an fMRI study. Biol Psychiatry. 2001; 50:651-58.

10. Zhong M, Wang X, Xiao J, Yi J, Zhu X, Liao J, Wang W, Yao S. Amygdala hyperactivation and prefrontal hypoactivation in subjects with cognitive vulnerability to depression. Biol Psychol. 2011; 88:233-42.

11. Jaworska N, Yang XR, Knott V, MacQueen G. A review of fMRI studies during visual emotive processing in major depressive disorder. World J Biol Psychiatry. 2015; 16:44871.

12. Samartzis L, Dima D, Fusar-Poli P, Kyriakopoulos M. White matter alterations in early stages of schizophrenia: a systematic review of diffusion tensor imaging studies. J Neuroimaging. 2014; 24:101-10.

13. Mueller S, Keeser D, Reiser MF, Teipel S, Meindl T. Functional and structural MR imaging in neuropsychiatric disorders, part 2: application in schizophrenia and autism. AJNR Am J Neuroradiol. 2012; 33:2033-37.

14. Vita A, De Peri L, Deste G, Sacchetti E. Progressive loss of cortical gray matter in schizophrenia: a meta-analysis and meta-regression of longitudinal MRI studies. Transl Psychiatry. 2012; 2:e190.

15. De Peri L, Crescini A, Deste G, Fusar-Poli P, Sacchetti E, Vita A. Brain structural abnormalities at the onset of schizophrenia and bipolar disorder: a meta-analysis of controlled magnetic resonance imaging studies. Curr Pharm Des. 2012; 18:486-94.

16. Vita A, De Peri L. The effects of antipsychotic treatment on cerebral structure and function in schizophrenia. Int Rev Psychiatry. 2007; 19:429-36.

17. Vita A, Dieci M, Giobbio GM, Tenconi F, Invernizzi G. Time course of cerebral ventricular enlargement in schizophrenia supports the hypothesis of its neurodevelopmental nature. Schizophr Res. 1997; 23:25-30.

18. Bousman CA, Cropley V, Klauser P, Hess JL, Pereira A, Idrizi R, Bruggemann J, Mostaid MS, Lenroot R, Weickert TW, Glatt SJ, Everall IP, Sundram S, et al. Neuregulin-1 (NRG1) polymorphisms linked with psychosis transition are associated with enlarged lateral ventricles and white matter disruption in schizophrenia. Psychol Med. 2017 Aug 22. https://doi.org/10.1017/S0033291717002173. [Epub ahead of print].

19. Wang S, Zhan Y, Zhang Y, Lyu L, Lyu H, Wang G, Wu R, Zhao J, Guo W. Abnormal long- and short-range functional connectivity in adolescent-onset schizophrenia patients: A resting-state fMRI study. Prog Neuropsychopharmacol Biol Psychiatry. 2018; 81:445-51.

20. Strigo IA, Murray SB, Simmons AN, Bernard RS, Huang JS, Kaye WH. The clinical application of fMRI data in a single-patient diagnostic conundrum: classifying brain response to experimental pain to distinguish between gastrointestinal, depressive and eating disorder symptoms. J Clin Neurosci. 2017; 45:149-53.

21. Duan C, Cosgrove J, Deligiannidis KM. Understanding Peripartum Depression Through Neuroimaging: a Review of Structural and Functional Connectivity and Molecular Imaging Research. Curr Psychiatry Rep. 2017; 19:70.

22. Jiang T, Zhou Y, Liu B, Liu Y, Song M. Brainnetome-wide association studies in schizophrenia: the advances and future. Neurosci Biobehav Rev. 2013; 37:2818-35.

23. Zhao K, Liu H, Yan R, Hua L, Chen Y, Shi J, Lu Q, Yao Z. Cortical thickness and subcortical structure volume abnormalities in patients with major depression with and without anxious symptoms. Brain Behav. 2017; 7:e00754.

24. Lan MJ, Rubin-Falcone H, Motiwala F, Chen Y, Stewart JW, Hellerstein DJ, Mann JJ, McGrath PJ. White matter tract integrity is associated with antidepressant response to lurasidone in bipolar depression. Bipolar Disord. 2017; 19:444-49.

25. Dong D, Wang Y, Chang X, Chen X, Chang X, Luo C, Yao D. Common and diagnosis-specific fractional anisotropy of white matter in schizophrenia, bipolar disorder, and major depressive disorder: Evidence from comparative voxelbased meta-analysis. Schizophr Res. 2017 Jul 4. http:// dx.doi.org/10.1016/j.schres.2017.07.003. [Epub ahead of print].

26. Dong D, Wang Y, Chang X, Jiang Y, Klugah-Brown B, Luo C, Yao D. Shared abnormality of white matter integrity in schizophrenia and bipolar disorder: A comparative voxelbased meta-analysis. Schizophr Res. 2017; 185:41-50.

27. Li H, Chan RC, McAlonan GM, Gong QY. Facial emotion processing in schizophrenia: a meta-analysis of functional neuroimaging data. Schizophr Bull. 2010; 36:1029-39.

28. Delvecchio G, Sugranyes G, Frangou S. Evidence of diagnostic specificity in the neural correlates of facial affect processing in bipolar disorder and schizophrenia: a metaanalysis of functional imaging studies. Psychol Med. 2013; 43:553-69.

29. Gur RE, Loughead J, Kohler CG, Elliott MA, Lesko K, Ruparel K, Wolf DH, Bilker WB, Gur RC. Limbic activation associated with misidentification of fearful faces and flat affect in schizophrenia. Arch Gen Psychiatry. 2007; 64:1356-66.

30. Kohler C, Swanson CL, Gur RC, Mozley LH, Gur RE. Depression in schizophrenia: II. MRI and PET findings. Biol Psychiatry. 1998; 43:173-80.

31. Kohler C, Gur RC, Swanson CL, Petty R, Gur RE. Depression in schizophrenia: I. Association with neuropsychological deficits. Biol Psychiatry. 1998; 43:16572.

32. Huebl J, Brücke C, Merkl A, Bajbouj M, Schneider GH, Kühn AA. Processing of emotional stimuli is reflected by modulations of beta band activity in the subgenual 
anterior cingulate cortex in patients with treatment resistant depression. Soc Cogn Affect Neurosci. 2016; 11:1290-98.

33. Jaworska N, Yücel K, Courtright A, MacMaster FP, Sembo M, MacQueen G. Subgenual anterior cingulate cortex and hippocampal volumes in depressed youth: the role of comorbidity and age. J Affect Disord. 2016; 190:726-32.

34. Liu Y, Du L, Li Y, Liu H, Zhao W, Liu D, Zeng J, Li X, Fu Y, Qiu H, Li X, Qiu T, Hu H, et al. Antidepressant Effects of Electroconvulsive Therapy Correlate With Subgenual Anterior Cingulate Activity and Connectivity in Depression. Medicine (Baltimore). 2015; 94:e2033.

35. Philippi CL, Motzkin JC, Pujara MS, Koenigs M. Subclinical depression severity is associated with distinct patterns of functional connectivity for subregions of anterior cingulate cortex. J Psychiatr Res. 2015; 71:103-11.

36. Niida A, Niida R, Matsuda H, Motomura M, Uechi A. Analysis of the presence or absence of atrophy of the subgenual and subcallosal cingulate cortices using voxelbased morphometry on MRI is useful to select prescriptions for patients with depressive symptoms. Int J Gen Med. 2014; 7:513-24.

37. Addington D, Addington J, Patten S. Depression in people with first-episode schizophrenia. Br J Psychiatry Suppl. 1998; 172:90-92.

38. Heald A, Morris J, Soni SD. Characterisation of depression in patients with schizophrenia. Indian J Med Res. 2008; 127:544-50.

39. Moreno GL, Bruss J, Denburg NL. Increased perceived stress is related to decreased prefrontal cortex volumes among older adults. J Clin Exp Neuropsychol. 2017; 39:313-25.

40. Rich AM, Cho YT, Tang Y, Savic A, Krystal JH, Wang F, $\mathrm{Xu} \mathrm{K}$, Anticevic A. Amygdala volume is reduced in early course schizophrenia. Psychiatry Res. 2016; 250:50-60.
41. Zhang J, Tan Q, Yin H, Zhang X, Huan Y, Tang L, Wang $\mathrm{H}, \mathrm{Xu}$ J, Li L. Decreased gray matter volume in the left hippocampus and bilateral calcarine cortex in coal mine flood disaster survivors with recent onset PTSD. Psychiatry Res. 2011; 192:84-90.

42. Zhuo C, Zhu J, Wang C, Qu H, Ma X, Tian H, Liu M, Qin W. Brain structural and functional dissociated patterns in schizophrenia. BMC Psychiatry. 2017; 17:45.

43. Zhuo C, Zhu J, Wang C, Qu H, Ma X, Qin W. Different spatial patterns of brain atrophy and global functional connectivity impairments in major depressive disorder. Brain Imaging Behav. 2017; 11:1678-89.

44. Ren W, Lui S, Deng W, Li F, Li M, Huang X, Wang Y, Li T, Sweeney JA, Gong Q. Anatomical and functional brain abnormalities in drug-naive first-episode schizophrenia. Am J Psychiatry. 2013; 170:1308-16.

45. First M, Spitzer R, Gibbon M, Williams J. Structured Clinical Interview for DSM-IV Axis I Disorders (SCID-I). Clinician Version, Administration Booklet. American Psychiatric Pub. 2012.

46. Kay SR, Fiszbein A, Opler LA. The positive and negative syndrome scale (PANSS) for schizophrenia. Schizophr Bull. 1987; 13:261-76.

47. Rekhi G, Ng WY, Lee J. Clinical utility of the Calgary Depression Scale for Schizophrenia in individuals at ultrahigh risk of psychosis. Schizophr Res. 2017 Jul 13. https:// doi.org/10.1016/j.schres.2017.06.056. [Epub ahead of print].

48. Gardner DM, Murphy AL, O’Donnell H, Centorrino $\mathrm{F}$, Baldessarini RJ. International consensus study of antipsychotic dosing. Am J Psychiatry. 2010; 167:686-93. 